\title{
Automatic evaluation of speech impairment caused by wearing a dental appliance
}

\author{
Mariko Hattori $^{1}$, Yuka I. Sumita ${ }^{2}$, Hisashi Taniguchi ${ }^{2}$ \\ ${ }^{1}$ Clinics for Oral and Maxillofacial Rehabilitation, Tokyo Medical and Dental University, Tokyo, Japan \\ ${ }^{2}$ Department of Maxillofacial Prosthetics, Tokyo Medical and Dental University, Tokyo, Japan \\ Email: sasamfp@tmd.ac.jp
}

Received 4 August 2013; revised 4 September 2013; accepted 21 September 2013

Copyright (C) 2013 Mariko Hattori et al. This is an open access article distributed under the Creative Commons Attribution License, which permits unrestricted use, distribution, and reproduction in any medium, provided the original work is properly cited.

\begin{abstract}
In dentistry, speech evaluation is important for appropriate orofacial dysfunction rehabilitation. The speech intelligibility test is often used to assess patients' speech, and it involves an evaluation by human listeners. However, the test has certain shortcomings, and an alternative method, without a listening procedure, is needed. The purpose of this study was to test the applicability of an automatic speech intelligibility test system using a computerized speech recognition technique. Speech of $\mathbf{1 0}$ normal subjects, when wearing a dental appliance, was evaluated using an automatic speech intelligibility test system that was developed using computerized speech recognition software. The results of the automatic test were referred to as the speech recognition scores. The Wilcoxon signed rank test was used to analyze differences in the results of the test between the following 2 conditions: with the palatal plate in place and with the palatal plate removed. Spearman correlation coefficients were used to evaluate whether the speech recognition score correlated with the result of conventional intelligibility test. The speech recognition score was significantly decreased when wearing the plate $(z=-2.807, P=0.0050)$. The automatic evaluation results positively correlated with that of conventional evaluation when wearing the appliance $(r=$ $0.729, P=0.017)$. The automatic speech testing system may be useful for evaluating speech intelligibility in denture wearers.
\end{abstract}

Keywords: Prosthodontics; Maxillofacial Prosthodontics; Speech; Automatic Speech Recognition

\section{INTRODUCTION}

The orofacial region is a complex of organs, most of which play a role in speech production. In the field of dentistry, speech evaluation is important for the appropriate orofacial dysfunction rehabilitation. Because the teeth and alveolar ridge are speech articulators, speech ability can be impaired by dental disorders such as missing teeth or by maxillofacial disorders caused by surgical treatment affecting orofacial structures such as resection of tumors in bone and soft tissue like the palate or tongue. Prosthetic treatment can often be useful for speech rehabilitation in such patients [1]. However, placement of dental appliances can sometimes compromise speech when the appliances interfere with articulation. For example, consonants are often affected by placing a new denture, and the patient needs time to compensate for this change [2]. Therefore, the speech of patients rehabilitated with prosthesis should be carefully assessed and monitored.

In dentistry, the speech intelligibility test is often used to assess speech and involves evaluation of the patient's speech by human listeners [3-6]. The procedure is simple; only a recording device is needed to record the patient's speech and play it back to the listeners for evaluation. However, the test is associated with time costs because listeners must provide their time to complete the evaluation. Moreover, the result is also dependent on the listener's ability [7]. Consequently, speech evaluation methods that do not require subjective listening evaluations have been sought, and an automatic speech intelligibility testing system using a computerized speech recognition technique was previously developed $[8,9]$.

These new systems have been used to evaluate speech in maxillectomy patients, which enable patients to selfrecord utterances and the examiners to obtain test results without requiring the inputs from human listeners.

Significant correlations were found between the results of the automatic tests and that of the conventional test involving human listeners. These findings suggested that the systems used in the present study, which involve 
no listening procedure, may effectively identify speech disorders in patients with maxillofacial defects.

In this study, the applicability of one of the new systems was tested to evaluate speech in normal subjects when wearing a dental appliance - in this instance-a palatal plate [9]. If the system detected slight differences in articulation caused by wearing the plate, it suggested that the system can be used not only for detecting severe speech disorders in patients with maxillofacial defects but also for minor speech problems in those wearing dentures. Two null hypotheses were proposed: 1) no differences in the result of the automatic test between 2 conditions will be observed; with the palatal plate in place and with the palatal plate removed; and 2) the speech recognition score will not correlate with the result of the conventional intelligibility test.

\section{MATERIALS AND METHODS}

The experimental palatal prosthesis was fabricated using auto-curing methylmethacrylate resin (Palapress Vario clear; Heraeus-Kulzer, Tokyo, Japan). An automatic speech intelligibility test system and the convention method, involved human listening, were used to evaluate speech in 10 subjects. The results of the 2 tests were compared.

\subsection{Subjects}

Ten women (mean age, 26.5 years) who were native Japanese speakers with normal speaking and hearing abilities participated in this study. All possessed 28 or more teeth in continuous dental arches. A power analysis was conducted with $\alpha=0.05$ and power $(\beta)=0.80$. On the basis of the results of a pilot study, a sample size of 10 was found to be appropriate. Informed consent was obtained from each subject, verbally and in writing. The study protocol was approved by the Ethics Committee of the Faculty of Dentistry, Tokyo Medical and Dental University (Registration No. 576).

\subsection{Fabrication of Experimental Palatal Plate}

An impression of the upper jaw was acquired using a ready-made impression tray and irreversible hydrocolloid impression material (Algiace Z; DENTSPLY-Sankin K. K., Tokyo, Japan), and an experimental palatal plate with a thickness of $2.0 \mathrm{~mm}$ was then fabricated using autocuring methylmethacrylate resin (Palapress Vario clear; Heraeus-Kulzer). Before the speech sample recording, subjects tested their palatal plate to ensure adequate retention and stability.

\subsection{Automatic Speech Intelligibility Testing}

The automatic conversation intelligibility test system [9] was installed on a laptop (Vaio PCG-NV99E, Sony, Tokyo, Japan) with Windows XP operating system (Microsoft, Tokyo, Japan). The automatic conversation intelligibility test system consists of the following 2 applications: a conversation intelligibility test recorder and a conversation intelligibility test result viewer. Block diagrams of applications are shown in Figures $\mathbf{1}$ and $\mathbf{2}$.

The conversation intelligibility test recorder consists of a patient information input module, a control module, a voice-input module, a test-text display module, a voice data store processing module, and a test-data storage unit. The conversation intelligibility test result viewer consists of a test data file input module, a voice recognition module, a test result display module, a unit sentence playback module, and a continuous playback module. Voice data are sent to the voice recognition module, where the computerized speech recognition software ViaVoice ${ }^{\circledR}$ (IBM ViaVoice for Windows, Pro V10 Japanese version, ScanSoft, Tokyo, Japan) is used.

The test text to be read aloud was displayed on the computer monitor test screen to create speech samples. Recognition results and scores were displayed on the results screen. The number of speech segments was calculated and automatically shown as a percentage on the upper right corner of the results screen. This percentage

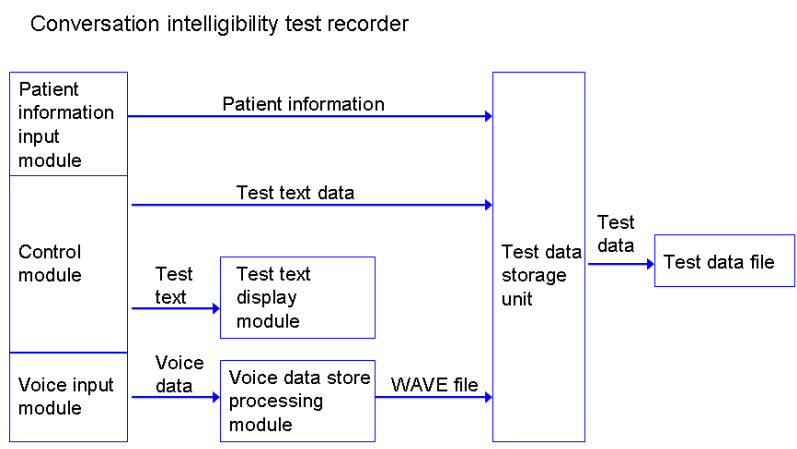

Figure 1. The block diagram of the conversation intelligibility test recorder.

Conversation intelligibility test result viewer

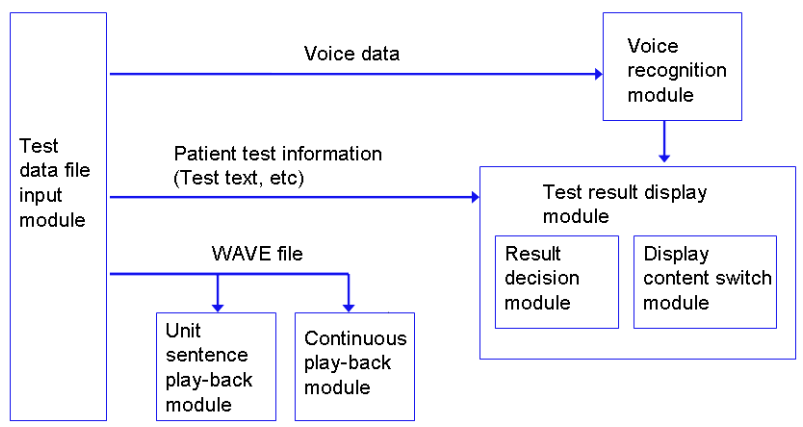

Figure 2. The block diagram of the conversation intelligibility test result viewer. 
constitutes the speech recognition score.

All 10 subjects were asked to speak the phrases shown one by one on the test screen. A microphone (F-VX400, Sony, Tokyo, Japan) was positioned $10 \mathrm{~cm}$ from the lips. Phrases from "Jack and the Beanstalk" were used as the test text. The utterances were recorded on the laptop computer by means of a sound interface (Sound Blaster Extigy, Creative Technology, Tokyo, Japan) in a soundproof room. The sampling rate was $11 \mathrm{kHz}$. The subjects' speech was tested under the following 2 conditions: with the palatal plate in place and with the palatal plate removed.

\subsection{Conventional Intelligibility Testing}

In a quiet room, the recorded utterances were played back on the computer to 5 linguistically-untrained adult listeners with no hearing impairment. The listeners evaluated each subject's utterances on a scale of 1 to 5 according to Terai's method for mild disorders as follows: 5, natural; 4, slightly unnatural; 3, sometimes unnatural; 2, slightly unintelligible; and 1, partially intelligible [6]. The mean of scores marked by the 5 listeners was used to calculate the test result, namely conversational intelligibility (Table 1).

\subsection{Statistical Analysis}

Normality was evaluated by normal distribution plots, and histograms were used to depict the variability. Nonparametric tests were applied to analyze the data. The Wilcoxon signed rank test was used to analyze differences in the speech recognition score and conversational intelligibility between the following 2 conditions: with the palatal plate in place and with the palatal plate removed. Conversational intelligibility and speech recognition score of all 10 subjects when wearing the palatal plate were plotted on a coordinate system. Spearman correlation coefficients were used to evaluate whether the speech recognition score correlated with conversational intelligibility. The analyses were conducted using the program Statview-J Version 5.0 (SAS Institute Inc., Cary, NC).

Table 1. The scale for the listening experiment of conversation intelligibility.

\begin{tabular}{cc}
\hline criteria & score \\
\hline natural & 5 \\
slightly unnatural & 4 \\
sometimes unnatural & 3 \\
slightly unintelligible & 2 \\
partially intelligible & 1 \\
\hline
\end{tabular}

\section{RESULTS}

Conversational intelligibility and speech recognition scores were not normally distributed with or without placement of the palatal plate. Thus, nonparametric tests were applied to analyze the data. The results of the automatic tests are shown in Figure 3; those of the conventional tests are shown in Figure 4.

The median and range of the speech recognition score for the 10 subjects were 67.6 and $53.8-75.3$ without the palatal plate and 53.1 and $0.0-66.1$ with the palatal plate, respectively. Thus, the speech recognition score was significantly decreased when wearing the plate $(\mathrm{z}=-2.807$, $\mathrm{P}=0.0050$; Figure 3).

All 10 subjects had a score of 5.0 in conversational intelligibility when not wearing the palatal plate. Median conversation intelligibility was 3.8 (range, 2.0 - 4.4) when wearing the plate, indicating a significant decrease in conversational intelligibility when wearing the palatal plate $(\mathrm{z}=-2.814, \mathrm{P}=0.0049$; Figure 4).

Speech recognition and conversational intelligibility scores showed a positive correlation when wearing the plate (correlation coefficient $(r)=0.729, P=0.017$; Figure 5).

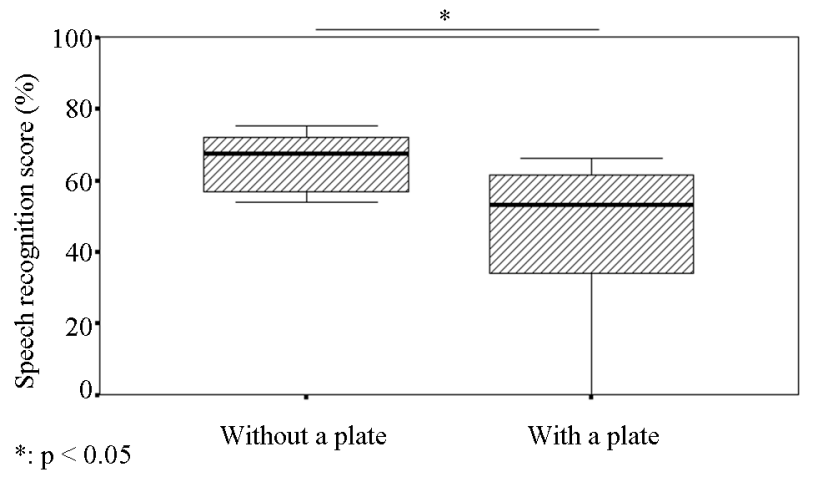

Figure 3. The results of the automatic tests. The speech recognition score was significantly decreased when wearing the palatal plate $(\mathrm{z}=-2.807, \mathrm{P}=0.0050)$.

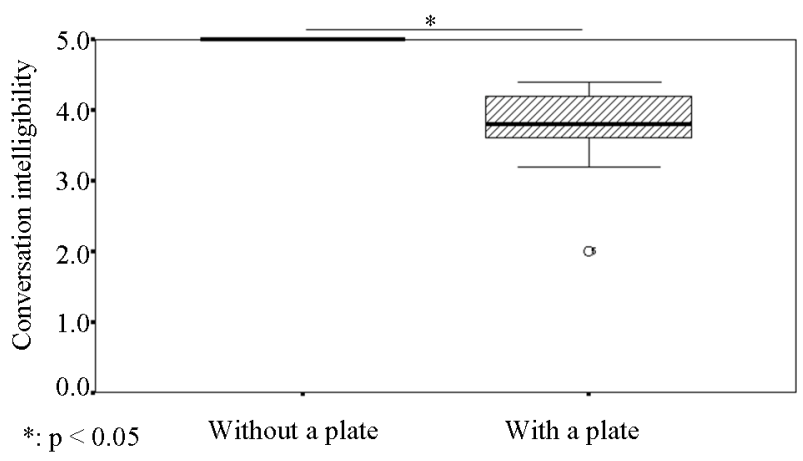

Figure 4. The results of the conventional tests. Conversational intelligibility was significantly decreased when wearing the palatal plate $(\mathrm{z}=-2.814, \mathrm{P}=0.0049)$. 


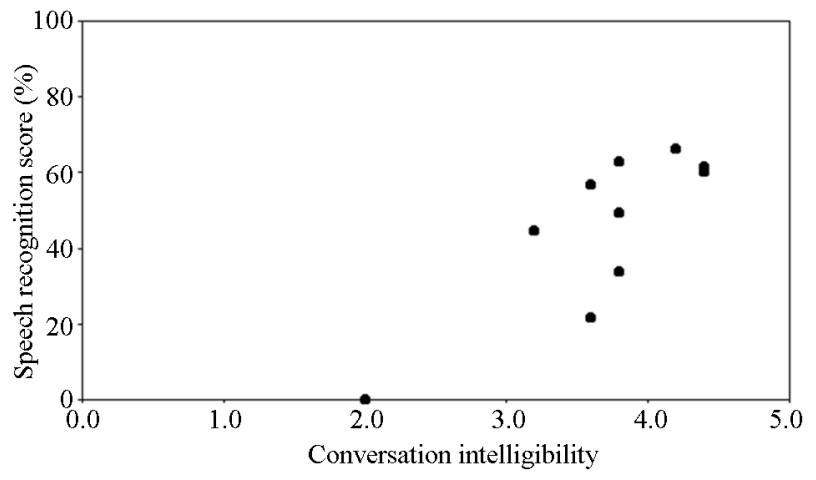

Figure 5. Speech recognition score and conversational intelligibility showed a positive correlation when wearing the plate (correlation coefficient $(r)=0.729, \mathrm{P}=0.017$ ).

\section{DISCUSSION}

The advantages of the automatic conversation intelligibility test system, which employs computerized recognition software based on a fast Fourier transform-based cepstrum analysis and hidden Markov model matching technology, are its easy operation, objective evaluation, and the lack of the need for human listeners [9]. In this study, the system was able to detect significant decreases in the speech recognition score when subjects were wearing the palatal plate, indicating that the system can detect slight differences in articulation caused by the plate.

Wearing the plate not only blocked sensory feedback from the palate but it also changed the positional relationship of the tongue and the articulators because of the plate's thickness. This affected consonant synthesis and resulted in an unnatural-sounding speech. Such an impairment created sufficient acoustic differences in some instances that the computerized speech recognition software recognized words that were different from the actual test words. This explains why the low conversational intelligibility scores given by human subjects in the traditional testing method correlated positively with the speech recognition score given by the automatic test system. In future, the automatic testing system may prove useful for evaluating the effects of different denture designs or of denture adjustment on speech.

The present study compared the speech recognition scores with and without wearing a dental appliance. Based on these results, the null hypotheses-there is no difference in the result of the automatic test between the following 2 conditions: with the palatal plate placed and with the palatal plate removed and speech recognition score is not correlated with the result of conventional intelligibility test-were rejected.

Future studies should examine the score using dental appliances of different designs. The results of computerized speech recognition are reported to be affected by variability in speaker characteristics such as age, sex, and accent $[10,11]$. Accordingly, the subjects in the present study were limited to same sex, had a small age distribution, and spoke 1 language in the same regional accent. Further study is warranted with a larger number of subjects of both sexes, in different age groups, and speaking with different accents. In addition, because utterances were recorded immediately after placing the palatal plate in this study, it will be interesting to examine changes in the speech recognition score over time as the subject gets habituated to the denture placement.

\section{CONCLUSION}

The automatic test system detected a significant difference in speech intelligibility, using the speech recognition score, in normal subjects with and without placement of a palatal plate. The results of the automatic test system and the conventional evaluation method by human listeners were positively correlated. These findings suggest that the system can be used to evaluate speech intelligibility of denture wearers.

\section{ACKNOWLEDGEMENTS}

This research was supported by the Japan Society for the Promotion of Science Grants-in-Aid for Scientific Research (KAKENHI; KAKENHI [Grant No. 23792207]).

This work was supported by a support program for women researchers from the Tokyo Medical and Dental University.

\section{REFERENCES}

[1] Chierici, G. and Lawson, L. (1973) Clinical speech considerations in prosthodontics: Perspectives of the prosthodontist and speech pathologist. Journal of Prosthetic Dentistry, 29, 29-39. http://dx.doi.org/10.1016/0022-3913(73)90136-4

[2] Kong, H.J. and Hansen, C.A. (2008) Customizing palatal contours of a denture to improve speech intelligibility. Journal of Prosthetic Dentistry, 99, 243-248. http://dx.doi.org/10.1016/S0022-3913(08)60049-9

[3] Umino, S., Masuda, G..G., Ono, S. and Fujita, K. (1998) Speech intelligibility following maxillectomy with and without a prosthesis: An analysis of 54 cases. Journal of Oral Rehabilitation, 25, 153-158. http://dx.doi.org/10.1046/j.1365-2842.1998.00238.x

[4] Rieger, J., Wolfaardt, J., Seikaly, H. and Jha, N. (2002) Speech outcomes in patients rehabilitated with maxillary obturator prostheses after maxillectomy: A prospective study. The International Journal of Prosthodontics, 15, 139-144.

[5] Sullivan, M., Gaebler, C., Beukelman, D., Mahanna, G., Marshall, J., et al. (2002) Impact of palatal prosthodontic intervention on communication performance of patients' maxillectomy defects: A multilevel outcome study. Head Neck, 15, 530-538. http://dx.doi.org/10.1002/hed.10095

[6] Terai, H. and Shimahara, M. (2001) Speech intelligibility 
in conversation of patients with mild articulatory disorders. Journal of the Japanese Stomatological Society, 50, 104-108.

[7] Imai, S., Yamashita, Y., Ohno, K., Suzuki, N. and Michi, K. (1994) Speech intelligibility in conversation of postsurgical oral cancer patients - Comparison with syllable intelligibility and patient's own assessment. The Japan Journal of Logopedics and Phoniatrics, 35, 29-38. http://dx.doi.org/10.5112/jilp.35.29

[8] Hattori, M., Sumita, Y.I., Kimura, S., Mukohyama, H. and Taniguchi, H. (2005) Development of an automatic speech intelligibility test system using a computerized speech recognition technique. Maxillofacial Prosthetics, 28, 36-48.
[9] Hattori, M., Sumita, Y.I., Kimura, S. and Taniguchi, H. (2010) Application of an automatic conversation intelligibility test system using computerized speech recognition technique. Journal of Prosthodontic Research, 54, 7-13. http://dx.doi.org/10.1016/j.jpor.2009.07.004

[10] Benzeghiba, M., De Mori, R., Deroo, O., Dupont, S., Erbes, T., et al. (2007) Automatic speech recognition and speech variability: A review. Speech Communication, 49 , 763-86. http://dx.doi.org/10.1016/j.specom.2007.02.006

[11] Rebman, C., Aiken, M. and Cegielski, C. (2003) Speech recognition in the human-computer interface. Information \& Management, 40, 509-519. http://dx.doi.org/10.1016/S0378-7206(02)00067-8 